(C) 2011 IEEE. Reprinted, with permission, from Brownsell, S., Bradley, D., Cardinaux, F. and Hawley, M. Developing a systems and informatics based approach to lifestyle monitoring within eHealth: part I- technology and data management. 1st IEEE Conference on Healthcare Informatics, Imaging and Systems Biology, San Jose, California, 26-29 July, 2011.

This material is posted here with permission of the IEEE. Such permission of the IEEE does not in any way imply IEEE endorsement of any of University of Abertay Dundee's products or services. Internal or personal use of this material is permitted. However, permission to reprint/republish this material for advertising or promotional purposes or for creating new collective works for resale or redistribution must be obtained from the IEEE by writing to pubs-permissionsdieee.org.

By choosing to view this document, you agree to all provisions of the copyright laws protecting it. 


\title{
Developing a Systems and Informatics based approach to Lifestyle Monitoring within eHealth: Part I- Technology and Data Management
}

\author{
Simon Brownsell, David Bradley, Fabien Cardinaux and Mark Hawley \\ ScHARR, University of Sheffield, \\ Regent Court, 30 Regent Street, \\ Sheffield S1 4DA, UK \\ e-mail: s.brownsell@sheffield.ac.uk
}

\begin{abstract}
Lifestyle monitoring forms a subset of telecare in which data derived from sensors located in the home is used to identify variations in behaviour which are indicative of a change in care needs. Key to this is the performance of the sensors themselves and the way in which the information from multiple sources is integrated within the decision making process. The paper therefore considers the functions of the key sensors currently deployed and places their operation within the context of a proposed multi-level system structure which takes due cognisance of the requisite informatics framework.
\end{abstract}

Keywords - Monitoring, Health information management, Machine intelligence, Public healthcare, Sensor systems

\section{INTRODUCTION}

Lifestyle monitoring systems constitute a sub-set within the wider and more general model of telecare which set out to provide information on a monitored individual's behaviour patterns. Behavioural changes over time that are indicative of a change in circumstances or care needs, will then trigger an appropriate alert enabling the requisite assistance to be provided.

Lifestyle monitoring, or behavioural monitoring as it is sometimes referred to, was effectively initiated by the 1995 study by Celler et al [1] which showed that an individual's health status could be determined by monitoring a number of relatively simple parameters that expressed the interaction between the individual and their local environment. The Celler study concluded that some $50 \%$ of individuals had undiagnosed medical problems that could be detected by home monitoring.

Follow on studies included that in the UK by the Anchor Trust and British Telecom who developed a system, which did not contain explicit medical data, to detect changes in a users lifestyle [2]. The key conclusions of this work was that:

- The system was generally acceptable.

- It enhanced feelings of safety and security in the home, reducing fears and apprehensions.

- It increased the care choices available.

- It supported and enhanced the carers role.

However, and despite growing interest in all aspects of telecare, few studies have actually sought to formally evaluate the performance of lifestyle monitoring systems, despite there being a number of commercial systems available and operation in a range of household environments $[3,4]$. Indeed, a review by the authors of the literature covering lifestyle monitoring [5] from 1990 to December 2009 identified, from an initial selection of 1,835 , some 74 papers of significance, of which only $4[6,7,8,9]$ were concerned with trials involving more than 20 subjects. A further 21 papers reported trials with fewer than 20 subjects, and in many cases only a single subject.

Such a weak formal evidence base, this despite the significant numbers of commercial installations around the world, means that the interpretation and analysis of the source data in relation to individual behaviour remains relatively problematic. Nor are there at present any formal or robust feedback mechanisms in place at the system level, or indeed any other level, to support an effective assessment of strategies. The role of such feedback mechanisms would be to take the evidence of outcomes resulting from the deployment and use of lifestyle monitoring data and hence use this to modify and enhance the performance of the interpretive algorithms being deployed. Thus, information from a particular individual could be used to enhance system behaviour in relation to a wider group of individuals having similar backgrounds and needs.

Indeed, in one of the 20 plus subject studies referred to above, that by Brownsell et al [9], it was concluded through retrospective analysis that sufficient and appropriate data would have been available to have automatically identified a person suffering from issues of malnutrition. However, other individuals who also required clinical interventions would not have been identified from the recorded data. Overall however, there were beneficial trends resulting from the deployment of the technology in terms of:

1. An increase in the amount of time spent out of the home.

2. Heightened feelings of safety during the day and night along with a reduced fear of crime.

However, work was required to:

(a) Match user and telecare technology requirements.

(b) Establish the effectiveness of automatic monitoring functions.

(c) Establish when to reliably escalate issues to health and care providers. 
TABLE I

LiFESTyle MONitoring SENSOR TECHNOLOGIES

\begin{tabular}{|lll|}
\hline & \multicolumn{2}{l|}{ Sensor or Monitored Parameter } \\
\hline PIR & Door OPEN/CLOSE & Drawer/Cupboard use \\
\hline Appliance use & Bed/Chair occupied & TV/Video use \\
\hline Telephone & Lighting & Temperature \\
\hline Flooding & Sink/Bath use & Fall detection \\
\hline & Gas (Flame) detection \\
\hline
\end{tabular}

TABLE II BETWEEN SELECTED STATUS INDICATORS, MEASURES FOR THESE AND ASSOCIATED DATA SOURCES

\begin{tabular}{|l|ll|}
\hline \multicolumn{1}{|c|}{ Indicator } & \multicolumn{1}{c|}{ Data Sources } \\
$\begin{array}{l}\text { Change in contact with health } \\
\text { services }\end{array}$ & - Observation \\
Change in meal preparation & - Activity (Room PIRs) \\
Change in sleeping patterns & - Activity (Room PIRs) \\
Change in time spent at home & - Refrigerator/cupboard use \\
Change in visitor numbers & - Activity (Room PIRs) \\
Change in washing/bathing & - Activity (Room PIRs) \\
Decline in personal care/ADL & - Observation \\
& - Activity (Room PIRs) \\
Dirty pots and dishes & - Chair/bed occupancy \\
General neglect of housework & - Activity (Room PIRs) \\
\hline
\end{tabular}

(d) Automatically adapt to changing user requirements

(e) Provide user feedback.

Turning again to the literature search referred to above, in terms of currently deployed technologies the Passive Infra-Red (PIR) motion detector remains the most commonly used form of sensor in lifestyle monitoring applications. These and other sensors and sensor applications identified from the literature are shown in Table I, excluding clinical monitoring systems and technologies such as heart rate and blood pressure monitors.

There also exists a range of indicators, such as those set out in Table II, which relate to and reference a change in an individual's status [10]. A key element of Table II that is not reflected in the literature review is the potentially significant role that observation has to play in establishing an individual's health status. The source of such observational data will typically be carers, family and friends, health visitors and general visitors, not all of whom are trained either in observation or reporting, yet whose information could be a significant contributor to the interpretation of the data provided by a range of sensors and sensing systems. The ability to capture and record observational data from all these sources is likely therefore to be a significant contributor to enhancing the effectiveness of future lifestyle monitoring systems, and hence their levels of acceptance.
The goal of lifestyle monitoring is, as has already been indicated, that of developing a model of individual behaviour using information derived from sensors and other sources and to detect deviations from that behaviour which are indicative of a change in need. However, the nature of the system, and variations between individuals, means that the data available for assessing system performance is generally limited. Most current lifestyle monitoring systems therefore tend to rely on a number of basic measures which can be interpreted either manually or semi-automatically, as for instance whether an individual has become active within a particular time frame. While this may be a valid approach for a significant number of individuals, for instance the 'well elderly', it is believed by the authors to be a limited strategy which may be difficult to expand to a wider range of individuals, particularly if potentially significant levels of intervention may be involved or required.

In any context however, it is clear that the ability to detect and respond to behavioural change is dependent upon the nature and quality, defined in terms of its precision and robustness, of the source data obtained from the sensors deployed within an individual's home environment.

The paper therefore proposes a formal system structure and hierarchy, the underlying rationale of which is to provide the basis of a predictive approach to lifestyle monitoring and discusses this structure taking account of current, and likely future, system technologies. Specifically, the paper considers the functions and operation of the lower three levels of this structure, encompassing the key sensors and sensing components for lifestyle monitoring and reports on scenario and other testing of current sensors to establish their operational characteristics. It then reviews developments in sensor technology which are likely to impact upon the way in which next generation lifestyle monitoring systems evolve.

Consideration of the upper two levels of the system model, dealing with Data Analysis and Interpretation and System and Information Management considerations respectively, are then the subject of a companion paper [11].

\section{SySTEM STRUCTURE}

Key to effective operation is the requirement that accurate and relevant data is provided for decision making. If at this fundamental level errors are present or evident, then this will inhibit the ability to make decisions. The system should therefore continually test itself to ensure that it is working correctly and that the data received is valid and meaningful.

The issue as to whether data should be analysed in the home or remotely is a matter of continuing discussion and debate. Assuming a high speed data link is available and appropriate data security is in place with overall system costs, both capital and revenue, at a similar value for each approach, then the decision as to where data should be analysed could be regarded as a matter of choice. However, wherever the analysis of the generated data is carried out, it is important that this takes place within a time frame consistent with achieving the desired level of response. 


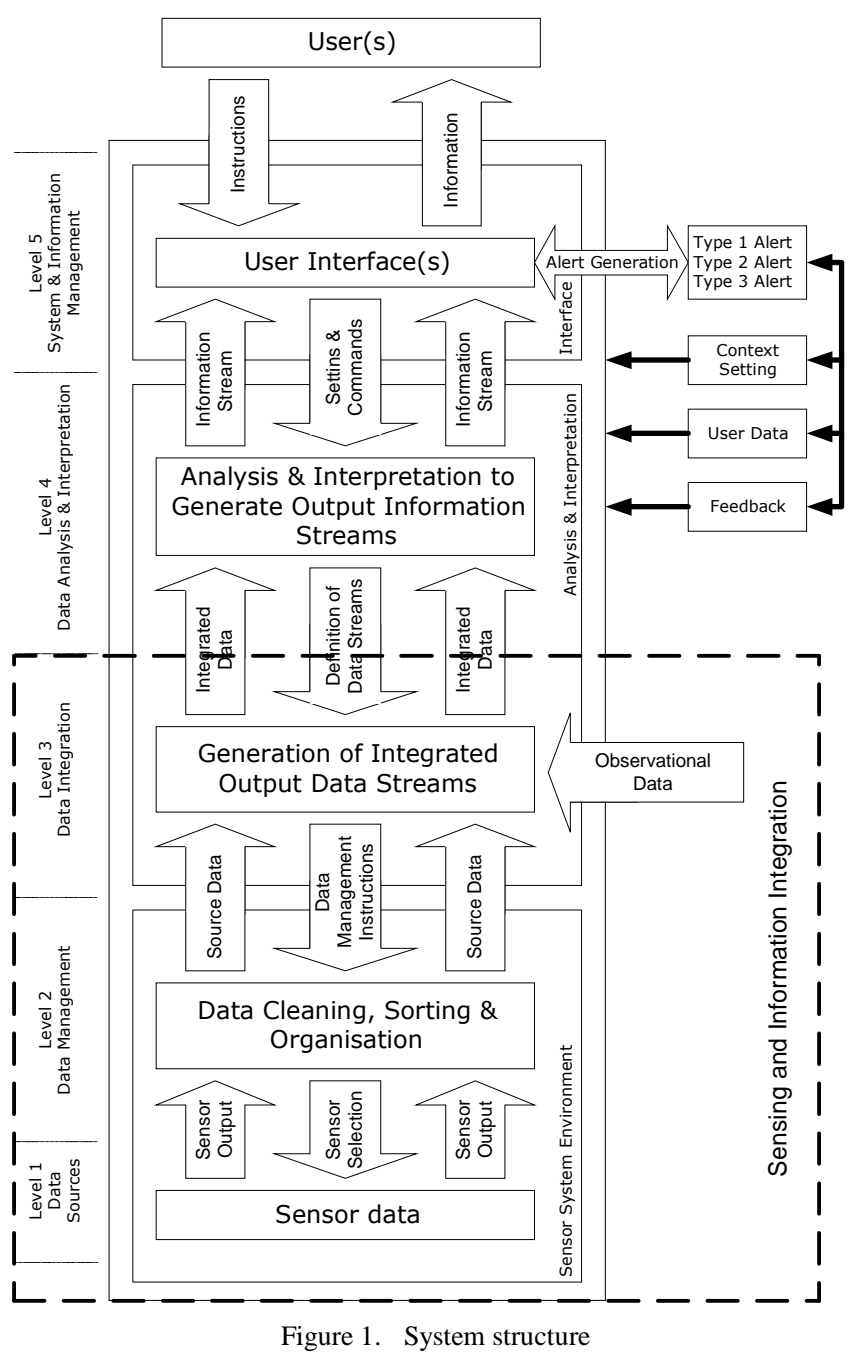

It can further be argued that inherent to the concept of a lifestyle monitoring system is a 'duty of care' to maximise user and clinical outcomes and that having data available that would result in an alert occurring but which is waiting to be downloaded for analysis is a less than optimal position. Hence in situations where a high speed data link is not available, it may be that a hybrid solution is required in which analysis is conducted in the home for immediate or short term alerts. Then for the detection of longer term conditions, the data may be downloaded once a day for analysis.

Figure 1 shows the proposed system structure to be associated with a lifestyle monitoring approach based on a combination of sensor and observational data. This structure was derived in particular from an analysis of the results of the Brownsell trials referred to earlier [9] and takes into account feedback from stakeholders as derived from focus group studies as well as the results of the literature survey [5] already referred to.

Referring to this figure it can be seen that it is structured around 5 operational levels together with alert generation and management. Of these five levels, this paper concerns itself with the lower 3 levels dealing with the capture of the source data, its management and the subsequent generation of the integrated and structured output data streams required for analysis and interpretation.

\section{LEVEL 1 - DATA SOURCES}

This level represents the mechanisms for data generation within the lifestyle monitoring environment. Data derived from the installed sensors will thus be time stamped and associated with a time frame referenced to both the individual and the nature of the data and available in the form of a time sequence. Observational data is however unlikely to be associated with a specified time frame and will instead form a part of the reference framework within which the sensor data is to be interpreted.

For the purposes of the paper the emphasis is on the use of sensors distributed throughout the home environment and excludes worn sensors. However, it should be noted that there is significant potential to link a lifestyle monitoring system based on combining distributed sensors with other monitoring strategies, as for instance those based around the use of body hubs $[12,13,14]$.

Table I presented earlier identified the main sensors identified in the literature as being associated with lifestyle monitoring. Of these, PIRs were the most common followed by door/cupboard OPEN/CLOSE, appliance use and chair/bed occupancy and these were therefore the subject of detailed analysis and laboratory and home based testing, including the use of the purpose built laboratory facility of Fig. 2.

PIR based motion detection - Three sets of conditions are considered in relation to the operation of PIR motion sensors, defined here as Type 1 operation, Type 2 operation and Type 3 operation respectively. Issues such as the overlap that might result during a transition between rooms will be excluded from this discussion. It should also be noted that the

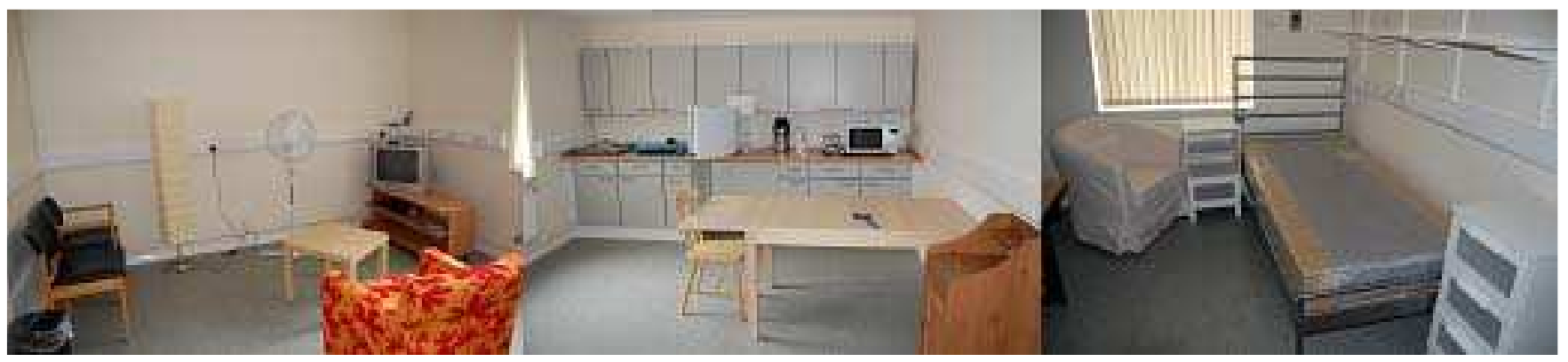

Figure 2. Laboratory environment 


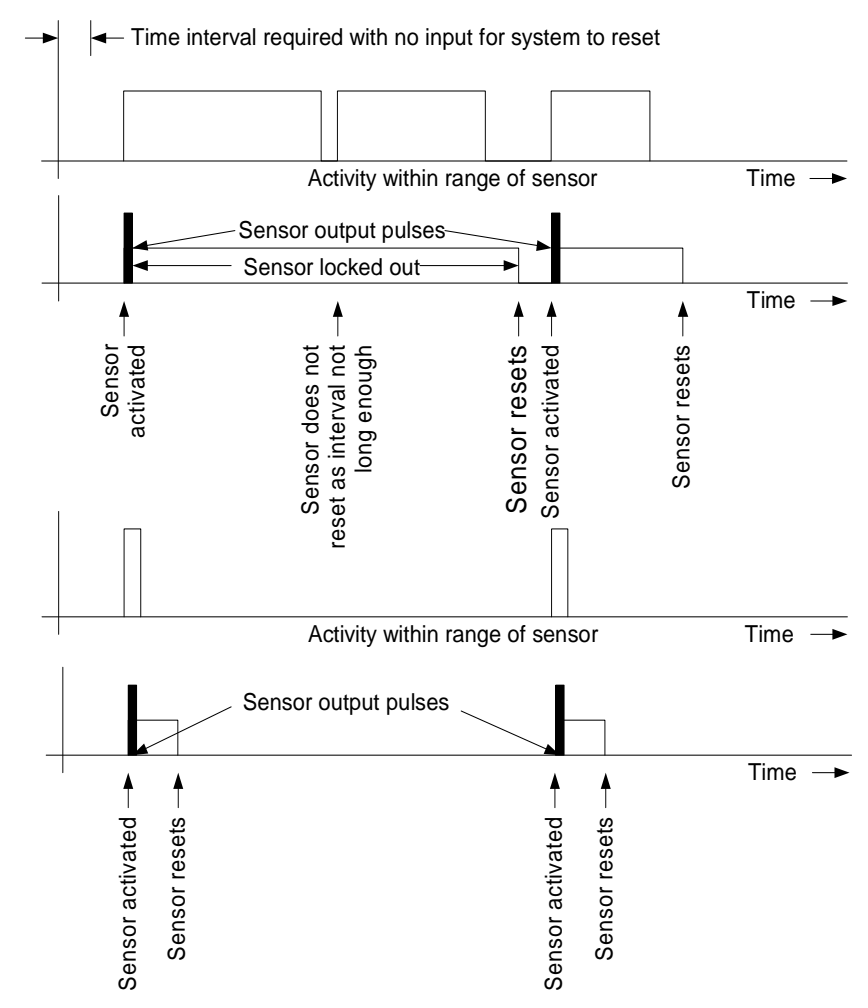

Figure 3. PIR Type 1 operation

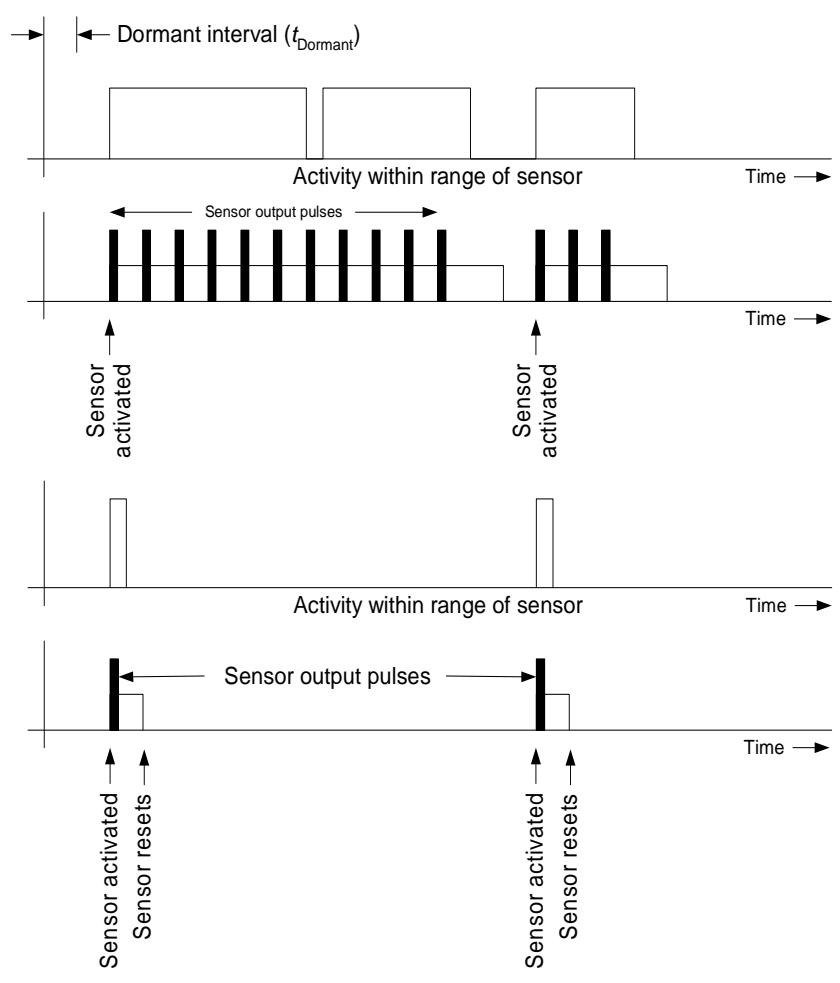

Figure 4. PIR Type 2 operation

discussion relates to single occupancy of the spaces to be monitored and that multiple occupancy would require an additional sensory layer to support discrimination between those sharing the space.

Type 1 Operation - To extend battery life, many commercially available wireless PIRs incorporate a reset period, often of several minutes, during which it is a requirement that no activity is detected at their input before they are able to transmit a further activation signal. Thus, once activated by movement, no further signal will be transmitted until such movement has ceased for an interval of at least the reset period. Once reset, any new movement which activates the device will transmit a signal, after which the reset requirement is re-established. This condition is illustrated by Fig. 3 .

From this it is apparent that in this mode of operation it is not possible to distinguish between a period of relatively continuous motion within the view of the sensor and a period in which no activity occurred. Such periods if inactivity could result from the monitored individual sitting in a chair, napping or even falling, yet under such conditions they would generate an output sequence no different from that for near continuous motion. This means that when operating in this mode, the sensor can be used to detect entry into a room, but not the level of activity, if any, within that room. Further, it cannot detect the exit from a room which can only be inferred by the activation of a sensor in a different room.

Thus in this mode it would only be suitable for lifestyle monitoring if what was required was a general indication of activity occurring over a relatively extended period of time. However, if a more detailed analysis of behaviour, and hence levels of activity, was the aim, then this mode of operation would suggest an unacceptable level of data loss.

Type 2 operation - In this mode of operation the sensor has a dormant period following each activation during which it resets itself. Once this dormant period ends, any motion detected will result in a new output. This condition is illustrated by Fig. 4 when for operation in this mode it is possible to discriminate between a period of near continuous activity and a period of inactivity in the same space.

Thus in this mode the PIR can be used to establish not only occupancy of space as for Type 1 operation, but also to monitor the general levels of activity and inactivity within that space. However, in this mode the PIR generates a continuous stream of pulses in the presence of activity, creating a large volume of data to be stored and manipulated to extract the required information.

Type 3 operation - In this mode the sensor has the same internal settings as for Type 1 . However in this case the sensor generates an output both when it is activated and when it resets. This mode of operation is shown in Fig. 5.

When operated in this mode, the PIR can be used to monitory occupancy of space, as for Type 1 operation, but can also be used to monitor the general level of activity or inactivity within a defined space as for Type 2 operation but without the accompanying volumes of data.

Detection envelope - The detection envelope for a typical PIR is of the order of $110^{\circ}$ in the horizontal plane and $90^{\circ}$ in the vertical plane. This means that if positioned correctly within a typical rectangular room, i.e. in a corner with the 


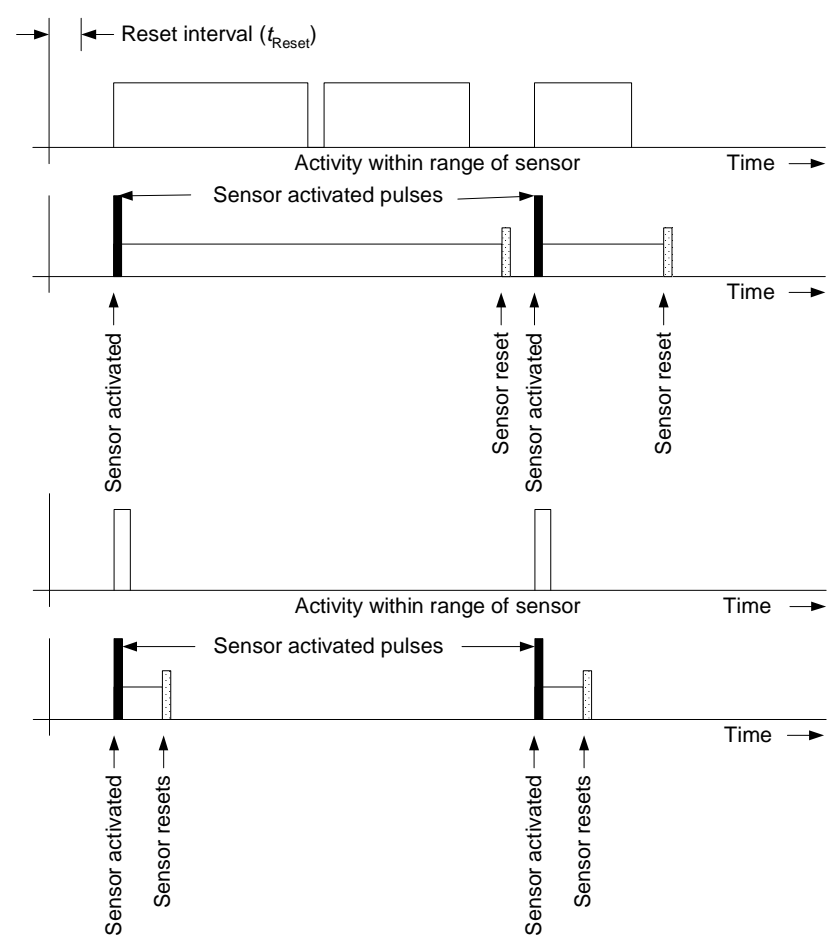

Figure 5. PIR Type 3 operation

outer edge of the envelope overlapping the shortest wall and tilted down at $45^{\circ}$, then the sensor should be able to detect any motion in the room out to its effective range ${ }^{1}$. Positioning must also take account of room shape (it is possible to use a correctly positioned sensor to cover an ' $\mathrm{L}$ ' shaped room) and of potential interference such as that would arise if a sensor was 'looking through' a door into a space covered by another sensor.

Electrical (appliance) sensors - These typically generate a signal which will allow for the time which the appliance is on to be determined. This may be (1) in the form of a continuous signal whilst the appliance is turned on or (2) a single pulse (SET) on turning on the appliance followed by a second pulse (RESET) when the appliance is turned off. In either case, the intent is to establish when and for how long appliances such as kettles, televisions, electric cookers and ovens of all types and other electrical appliances are used.

However, such sensors are typically associated with a specific socket rather than a specific appliance, which can mean that it is difficult to interpret the resulting data if the socket is used for a number of appliances or an appliance is moved from socket to socket. Other problems include using a socket in association with an extension cable which is then used to supply several appliances.

Bed and chair occupancy sensors - These typically generate a signal when the chair or bed is first occupied and the appropriate signal status is then maintained until occupancy ceases. It is recognised that this does not accommodate the ability to detect and respond to smaller movements whilst either sleeping or sitting. This could result

\footnotetext{
1 Taken here to be the maximum range at which detection is guaranteed.
}

in multiple activations when, for instance, a person is adjusting their seating position.

A particular problem in the design of chair sensors is that of making them compatible with a wide range of chair configurations. In general, the 'one size fits all' approach based on pressure pads has proved to be unsatisfactory and a new approach to the sensing of chair occupancy is probably required in order to further develop lifestyle reassurance and monitoring on the basis of robust and reliable data from this source.

Door Sensors - These tend to be either mechanically or magnetically actuated switches to detect if a door is opened or closed. Issues associated with these include contact bounce, resulting in apparent multiple operations in a short time interval and the effect of partial or incomplete closure of the door. The former condition can be dealt with be the inclusion either of appropriate anti-bounce circuitry or in software and part of the data cleaning process. However, partial or incomplete closure may not necessarily be detected, resulting in false indicators.

\section{A. Sensor testing protocols}

In order to characterise the various sensors described above, a series of tests structured around the core elements of a commercially available system and focused on repeated sensor activation over an extended time period was carried out to establish a baseline performance. Each sensor was tested individually, for instance by repeatedly turning $\mathrm{ON}$ and OFF the appliance for an electrical sensor or OPENING and CLOSING a door or cupboard. The following protocols were adopted to support the performance evaluation.

Individual sensor testing - The actions required to activate each type of sensor in the dwelling were performed with a predefined timing. This served to identify specific issues such as contact bounce associated with the operation of door and cupboard sensors, enabling remedial measures to be developed.

Adverse conditions testing - This required actions to be performed to generate activation errors, as for instance the simultaneous activation of two or more sensors.

False activation testing_- This is aimed at verifying that no sensor activation occurs at periods when it is known that no-one is in the laboratory environment of Fig. 2.

Controlled scenarios - A set of scenarios or scripts structured around activities of daily living and which defined precise sequence and timing of actions to be performed in the dwelling. This protocol forms part of the reliability test and also further evaluates sensor performance. The scenarios were performed several times in the laboratory and test users were then asked to perform the same tasks in their own homes.

Weakly controlled scenarios - These were similar to the controlled scenario protocols, but with an undefined series of actions. Thus, an individual is asked to simulate the preparation of a meal but without specifying exactly what actions to perform or in which sequence. Individuals were asked to record in a diary or log when they are performing certain activities. 


\section{B. Sensor testing results}

Along with Fig. 6, Tables III to VI provide the results obtained from the sensor testing procedures. Table VII then presents a summary of the tests results showing for each sensor type the number and rate of missed actions and the number of spurious or unexpected events in the data file.

It was noted that while some of the spurious events and missed actions were due to problems related to the sensors, as for instance contact bounce which could potentially be eliminated at the data cleaning stage, some are likely to be

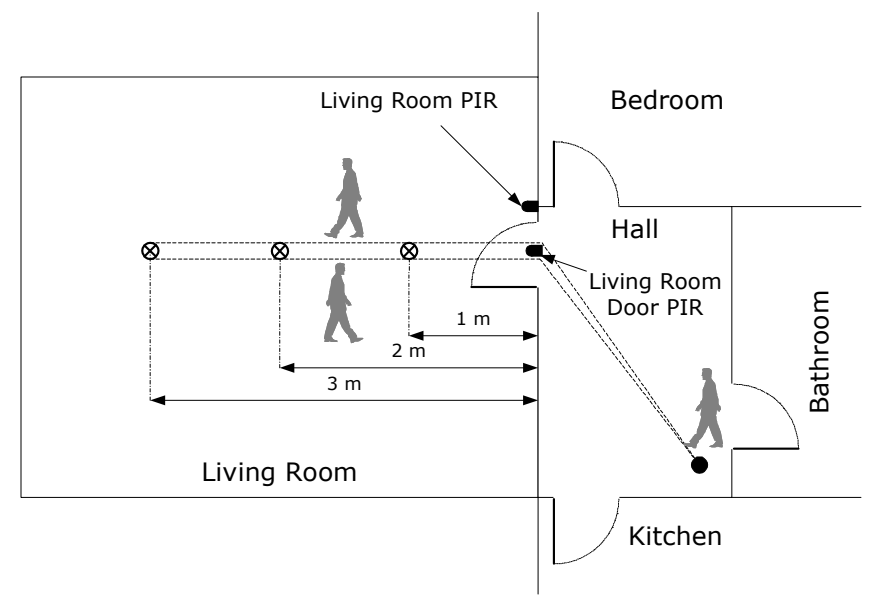

Figure 6. Schematic of test environment showing PIR positions and target points

TABLE III _ . PIR Test RESUlts

\begin{tabular}{|l|r|r|r|r|}
\hline \multirow{2}{*}{ Sensor } & \multicolumn{2}{|c|}{$\begin{array}{c}\text { Session 1 } \\
\text { Individual 1 - 'Tall' }\end{array}$} & \multicolumn{2}{|c|}{$\begin{array}{c}\text { Session 2 } \\
\text { Individual 1 - 'Tall' }\end{array}$} \\
\cline { 2 - 6 } & Missing & Spurious & Missing & Spurious \\
\hline $\begin{array}{l}\text { Living room door } \\
\text { PIR }\end{array}$ & $0 / 30$ & 3 & $0 / 30$ & 0 \\
\hline Living room PIR & $0 / 15$ & 4 & $0 / 15$ & 0 \\
\hline PIR Bedroom & - & 15 & & \\
\hline Total & $0 / 45$ & 21 & $0 / 45$ & 0 \\
\hline Sensor & $\begin{array}{r}\text { Session 3 } \\
\text { Individual 2 - } \\
\text { 'Average' }\end{array}$ & $\begin{array}{r}\text { Session 4 } \\
\text { Individual 2 - } \\
\text { 'Average' }\end{array}$ \\
\hline $\begin{array}{l}\text { Living room door } \\
\text { PIR }\end{array}$ & $0 / 30$ & 0 & $0 / 30$ & 0 \\
\hline Living room PIR & $0 / 15$ & 0 & $0 / 15$ & 0 \\
\hline Total & $0 / 45$ & 0 & $0 / 45$ & 0 \\
\hline
\end{tabular}

TABle IV Appliance Sensor Test Results

\begin{tabular}{|l|r|r|}
\hline & Missing & Spurious \\
\hline Lamp session 1 & $7 / 25$ & 0 \\
\hline Lamp session 2 & $0 / 25$ & 0 \\
\hline Kettle session 1 & $0 / 25$ & 0 \\
\hline Kettle session 2 & $0 / 25$ & 0 \\
\hline Total & $7 / 100$ & 0
\end{tabular}

associated with the platform. Indeed, on occasion no outputs were generated for periods of several minutes, suggesting that missing actions and spurious events could be associated with platform operation.

There were also a very high number of instances when sensors were activated simultaneously, which resulted in missed actions. During these tests, PIRs were 'blinded' when not in use to ensure that only sensors involved in the tests would generate data. In a 'real world' environment, more simultaneous activations would be anticipated.

Following on from the testing and evaluation performed in the laboratory, a set of scripts such as those shown in Table VIII were developed to be performed by the user participants in their own home. These scripts were adapted as appropriate to take account of factors such as the participants physical capacity and capability, the equipment installed in their home and its configuration. Before asking any participant to perform a script, the researchers ensured that they were willing to do so and that they did not have any impairment that would prevent them from doing so in a safe manner. Appropriate ethical approvals were also obtained.

The installation for each participant was adapted with regard to their preferences, furniture and appliances in their house. The total number of sensors was also limited in order to avoid conflicts caused by simultaneous activation. In particular, those sensors such as the appliance sensor which proved to be most unreliable when tested in the laboratory

Table V. Chair and Bed SENSOR Test Results

\begin{tabular}{|l|r|r|}
\hline & Missing & Spurious \\
\hline Chair user 1 & $12 / 25$ & 0 \\
\hline Chair user 2 & $12 / 25$ & 0 \\
\hline Bed user 2 & $0 / 25$ & 3 \\
\hline Total & $24 / 75(32 \%)$ & 3 \\
\hline
\end{tabular}

Table VI. Simultaneous actuivation Test Results

\begin{tabular}{|l|r|r|}
\hline & Missing & Spurious \\
\hline Session 1 & $24 / 25$ & 0 \\
\hline Session 2 & $16 / 25$ & 0 \\
\hline Total & $40 / 50(80 \%)$ & 0 \\
\hline
\end{tabular}

TABLE VII. OVERALL ERRORS

\begin{tabular}{|l|r|r|}
\hline & Missing & Spurious \\
\hline Electrical (power) sensors & $9 / 130(7 \%)$ & 16 \\
\hline $\begin{array}{l}\text { Door/Drawer open/close } \\
\text { (individually) }\end{array}$ & $10 / 160(6 \%)$ & 4 \\
\hline Bed/Chair occupancy & $25 / 97(26 \%)$ & 6 \\
\hline PIR & $9 / 440(2 \%)$ & 61 \\
\hline $\begin{array}{l}\text { Door/Drawer open/close } \\
\text { (simultaneous activations) }\end{array}$ & $40 / 50(80 \%)$ & 0 \\
\hline Total & $133 / 877(15 \%)$ & 87 \\
\hline
\end{tabular}


TABLE VIII $\quad$ EXAMPLE SCRIPTS

\begin{tabular}{|c|c|}
\hline Script 1 & Script 2 \\
\hline 1. Start outside kitchen & 1. Start outside kitchen \\
\hline 2. Wait $1 \mathrm{~min}$ & 2. Wait $1 \mathrm{~min}$ \\
\hline 3. Walk into the kitchen & 3. Walk into the kitchen \\
\hline 4. Turn on the kettle & 4. Open cupboard \\
\hline 5. Open the fridge & 5. Wait $5 \mathrm{~s}$ \\
\hline 6. Wait $5 \mathrm{~s}$ & 6. Close cupboard \\
\hline 7. Close the fridge & 7. Open drawer \\
\hline 8. Turn off the kettle & 8. Wait $5 \mathrm{~s}$ \\
\hline \multirow[t]{5}{*}{ 9. Leave the room } & 9. Close drawer \\
\hline & 10. Open cupboard \\
\hline & 11. Wait $5 \mathrm{~s}$ \\
\hline & 12. Close cupboard \\
\hline & 13. Leave kitchen \\
\hline
\end{tabular}

were not installed. Participants were then asked to perform the set of 7 scripts once a day in weeks 1 and 6 of the trial period, and were asked to keep a diary of actions during that period.

The results of the home based trials are consistent with those obtained in the laboratory with an average of $14.4 \%$ missed events as opposed to $15 \%$ in the laboratory and 9 spurious events for the $90(10 \%)$ expected events as opposed to 87 spurious events against 877 (9.9\%) expected in the laboratory.

\section{LEVEL 2 - DATA MANAGEMENT}

At this level, data from the individual sources is validated and organised to form the source data streams. Thus at this level the data from a door (cupboard) open/close sensor would be checked for any evidence of multiple activations associated with 'bounce' and the information from PIR sensors would be referenced against the appropriate time slots making up the day. Consistency with the data requests transmitted from Level 3 is also checked before the request for data is transmitted to Level 1 and hence to the sensors themselves. In this context, this would not only include data associated with lifestyle monitoring but also with a change in mode or role, as for instance from monitoring to security when the occupant leaves their house or flat.

\section{LEVEL 3 - DATA INTEGRATION}

At this level, the source data streams are combined to generate the integrated data streams to be used for the analysis and interpretation of individual behaviour. In illustration of this, consider the need to generate information regarding the periods of occupancy of a particular room during a defined period of time $T_{\text {Daytime }}$ such that:

$T_{\text {Daytime }}=T_{\text {Day.End }}-T_{\text {Day.Start }}$

When:
IF Time IS During. $T_{\text {Daytime }}$ AND Outside.Flag IS NOT SET

THEN Establish.Space.Occupancy

FOR Each.Space.of.Interest

THEN FOR Each.Time

IF Activity.Detected

THEN Room.Occupancy.Flag IS SET

IF No.Activity.Detected

END FOR LOOP

THEN Room.Occupancy.Flag IS NOT SET

\section{END FOR LOOP}

END IF

If Outside.Flag is SET, this means that the occupier has left the house or flat, PIRs could then be operated in a security mode to detect intruders.

It is also at Level 3 that observational data would be entered and integrated with the data from the physical sensors. The integrated data streams are then transmitted to system Level 4 for analysis and interpretation.

\section{IMPACT OF TECHNOLOGY}

For lifestyle monitoring systems to be effective they need to have the flexibility to rapidly adapt and respond to user need. Unfortunately, many of the current sensor forms do not offer the degree of flexibility required in order to provide the levels of response required, and moreover often require relatively specialist installation. With the advent of wireless networking, the opportunity exists to deploy a new range of self organising sensors which afford the possibility of enhanced data collection $[15,16]$.

These developments will impact on the way in which lifestyle monitoring systems are installed and operated. In essence, the availability of low cost sensing will increase the flexibility of the installations and will facilitate the generation of rich and comprehensive sets of behavioural data. Further, the availability of significant processing power at the level of the sensor means that it becomes possible to consider concepts of distributed processing to handle the increased levels of data, using the sensors themselves as the processing nodes for real-time data analysis linked directly to the individual's environment.

\section{CONCLUSIONS}

Based on the discussion in the paper, it is clear that the present generation of sensors and their mode of deployment supports an ability to provide a level of discrimination, as for instance associated with determining if there is some level of activity within the monitored environment over a defined period of time. However, they do not support particularly well or effectively the data requirements associated with the interpretation of behaviour in relation to identified indicators. A shift to a behaviour based approach to lifestyle monitoring and reassurance will therefore require a revised approach to monitoring based around a range of sensors and associated technology that support higher levels of discrimination than at present, and which are more flexible in both deployment and use than current generations of sensors. 
Where new generations of sensors are deployed, the data they generate creates new challenges in terms of data validation and analysis which must be addressed to enable the robust integration of outcomes with service provision to ensure a cost effective solution to the provision of support at the level of the individual. The advent of these new sensors also presents issues and problems for the testing and validation regimes to be used in association with them.

At present, each individual lifestyle monitoring installation represents a distinct and unique experiment in its own right based on an interpretation of potential behaviour rather than an understanding of actual behaviour. As a result, the algorithms being proposed are based on an essentially incomplete understanding of system behaviour, of the limitations of that behaviour and of the interaction with the monitored individual.

The detailed discussion of these interactions and the ways in which behavioural algorithms can be formulated is beyond the scope of this paper. Instead, as previously indicated, these topics are the subject of a companion paper [11] dealing with the upper two levels, Level 4 and Level 5, of the system structure of Fig. 1.

\section{ACKNOWLEDGEMENT}

The paper is based on independent research commissioned by the National Institute for Health Research in the UK. The views expressed in this publication are those of the author(s) and not necessarily those of the UK National Health Service, the National Institute for Health Research, or the UK Department of Health.

\section{REFERENCES}

[1] BG Celler, W Earnshaw, ED Ilsar, L Betbeder-Matibet, MF Harris, R Clark, T Hesketh and NH Lovell, "Remote monitoring of health status of the elderly at home. A multidisciplinary project on aging at the University of New South Wales", Int Biomed Comput, 40(2) 1995, pp 147-55

[2] S Brownsell and D Bradley (Ed J Porteous), "Assistive Technology and Telecare: Forging solutions for independent living", The Policy Press, 2003, ISBN 1-86134-462-7
[3] A Glascock A and D Kutzik, "The impact of behavioral monitoring technology on the provision of health care in the home". J. Univers. Comput. Sci.,12, 2006, 59-79

[4] A Sixsmith, N Hine, I Neild, N Clarke, S Brown and P Garner, "Monitoring the well-being of older people", Topics in Geriatric Rehabilitation, 23, 2007, pp 9-23

[5] S Brownsell, D Bradley, S Blackburn, F Cardinaux and MS Hawley, "A systematic review of lifestyle monitoring technologies", J. Telemedicine and Telecare, 17, pp 185 - 189

[6] NM Barnes, NH Edwards, DAD Rose and P Garner, "Lifestyle monitoring technology for supported independence", Computing and Control Engineering Journal, August1998, pp 169-174

[7] A Sixsmith, "An evaluation of an intelligent home monitoring system", J. of Telemedicine and Telecare, 6, 2000, pp 63-72

[8] M Alwan, S Dalal, D Mack, SW Kell, B Turner, J Leachtenauer and R Felder, "Impact of Monitoring Technology in Assisted Living: Outcome Pilot", IEEE Trans. Inf. Tech in Biomedicine, 10(1), 2006, pp 192-198

[9] S Brownsell, S Blackburn and MS Hawley, "An evaluation of second and third generation telecare services in older people's housing", J. Telemedicine and Telecare; 14, 2008, pp 8-12

[10] S. Brownsell, D. Bradley, S. Blackburn, F. Cardinaux and M.S. Hawley, "A review of behavioural monitoring methods and recommendations for future development", Int. Soc. of Gerontechnology, Gerontechnology, 2010, 9(2), page 20

[11] S Brownsell, DA Bradley, S Blackburn, F Cardinaux, \& MS Hawley, "Developing a Systems and Informatics based approach to Lifestyle Monitoring within eHealth: Part II - Analysis and Interpretation", IEEE Conf. on Healthcare Informatics, Imaging, and Systems Biology ( HISB 2011), 2011, Paper 27

[12] VM Jones, RGA Bults, D Konstantas and PAM Vierhout. "Body Area Networks for Healthcare", Wireless World Research Forum meeting, 2002, Stockholm, (http://doc.utwente.n1/66761/ - accessed $16^{\text {th }}$ September 2010)

[13] GE Churcher, G Bilchev, J Foley, R Gedge R and T Mizutani, "Experiences applying Sensor Web Enablement to a practical telecare application", $3^{\text {rd }}$ Intl. Symposium on Wireless Pervasive Computing, ISWPC 2008, pp 138 - 142

[14] M Mazzù, S Scalvini, A Giordano, E Frumento, H Wells, K Lokhorst and F Glisenti F, "Wireless-accessible sensor populations for monitoring biological variables", J Telemedicine \& Telecare, 14, 2008, pp 135-137

[15] //ubimon.doc.ic.ac.uk/bsn/index.php?m=206 (accessed $16^{\text {th }}$ September 2010)

[16] www.snm.ethz.ch/Main/HomePage (accessed $16^{\text {th }}$ September 2010) 